\title{
CARACTERIZAÇÃO DE PROPRIEDADES MECÂNICAS E ANISOTROPIA DE UMA CHAPA DE AÇO DP600*
}

\author{
Mateus Sotelo Menezes ${ }^{1}$ \\ Juliana Zottis ${ }^{2}$ \\ Alexandre da Silva Rocha ${ }^{3}$
}

\section{Resumo}

A família de aços avançados de alta resistência - AHSS contempla uma variedade de materiais que são utilizados principalmente na indústria automotiva. Os aços bifásicos incluem-se nesta classe e são assim denominados devido a sua microestrutura composta por ferrita e martensita. Apresentam alta resistência mecânica associada à ductilidade, o que permite a aplicação de grandes esforços em pequenas áreas sem que ocorra fratura. Este trabalho visa verificar, através de caracterização metalográfica, ensaios de tração e anisotropia, as propriedades mecânicas do aço DP600 para trabalho a frio. A curva tensão-deformação de engenharia para este aço foi estabelecida através de ensaios de tração até a ruptura do corpo de prova. Foram obtidos valores médios de $330 \mathrm{MPa}$ para o limite de escoamento, $589 \mathrm{MPa}$ para o limite de resistência à tração e $20 \%$ de alongamento. Os índices de anisotropia também foram verificados através de ensaios de tração. Estes resultados foram comparados com valores encontrados na literatura. De um modo geral, as chapas ensaiadas resultaram em uma diferença de valores de aproximadamente $10 \%$, possibilitando sua utilização a frio.

Palavras-chave: Aços bifásicos; DP600; Ensaio de tração; Anisotropia.

\section{CHARACTERIZATION OF MECHANICAL PROPERTIES AND ANISOTROPY OF A DP600 STEEL SHEET}

\section{Abstract}

The AHSS family includes a variety of materials that are mainly used in automotive industry. Dual Phase steels are included in this class and they are so named because of its microstructure consist of ferrite and martensite. These steels have high strength combined with ductility, which allows the application of large stress in small areas without fracture occurs. This study aims to verify by metallographic characterization, tensile test and anisotropy, the mechanical properties of DP600 steel for cold working. The engineering stress-strain curve to this steel has been established by tensile tests to rupture of the test specimen. Were obtained average values to yield strength of $330 \mathrm{MPa}$, to tensile strength of $589 \mathrm{MPa}$ and a total elongation of $20 \%$. The anisotropy numbers were also verified by tensile tests. These results were compared with values found in the literature. In general, the tested sheets resulted in a difference of values of about $10 \%$ allowing its use in cold working.

Keywords: Dual Phase steels; DP600; Tensile test; Anisotropy.

Aluno de graduação em engenharia metalúrgica, Laboratório de Transformação Mecânica, Universidade Federal do Rio Grande do Sul, Porto Alegre, RS, Brasil.

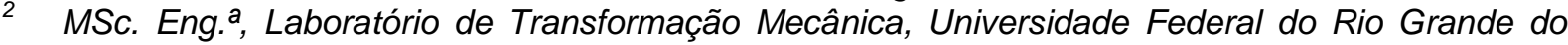
Sul, Porto Alegre, RS, Brasil.

3 Prof. Dr. Eng., Laboratório de Transformação Mecânica, Universidade Federal do Rio Grande do Sul, Porto Alegre, RS, Brasil. 


\section{INTRODUÇÃO}

\subsection{Aços Avançados de Alta Resistência - AHSS}

A indústria automobilística é a principal responsável pelos avanços tecnológicos dos aços nos últimos anos. Os primeiros estudos, ainda na década de 70, enfatizavam a utilização de elementos de liga como $\mathrm{Ni}$, $\mathrm{Ti}$ e $\mathrm{V}$ para proporcionar um aumento de resistência mecânica. Nos anos que seguiram, a ideia de uma estrutura bifásica foi implantada, objetivando reduzir as perdas de estampabilidade decorrentes dos maiores níveis de resistência mecânica alcançados [1].

A classe de aços avançados de alta resistência - AHSS (Advanced High Strenght Steel), assim conhecida atualmente, inclui, entre outros, os seguintes aços:

- Aços bifásicos - DP (Dual Phase);

- Aços martensíticos - MS;

- Aços de fase complexa - CP (Complex Phase);

- Aços com plasticidade induzida por transformação - TRIP (TransformationInduced Plasticity);

- Aços com plasticidade induzida por maclação - TWIP (Twinning-Induced Plasticity).

A Figura 1 permite uma comparação entre as propriedades mecânicas dos aços avançados de alta resistência e os aços convencionais. $O$ aumento da resistência mecânica é acompanhado de redução no alongamento e a utilização de microestruturas adequadas permite que esse efeito seja reduzido [2].

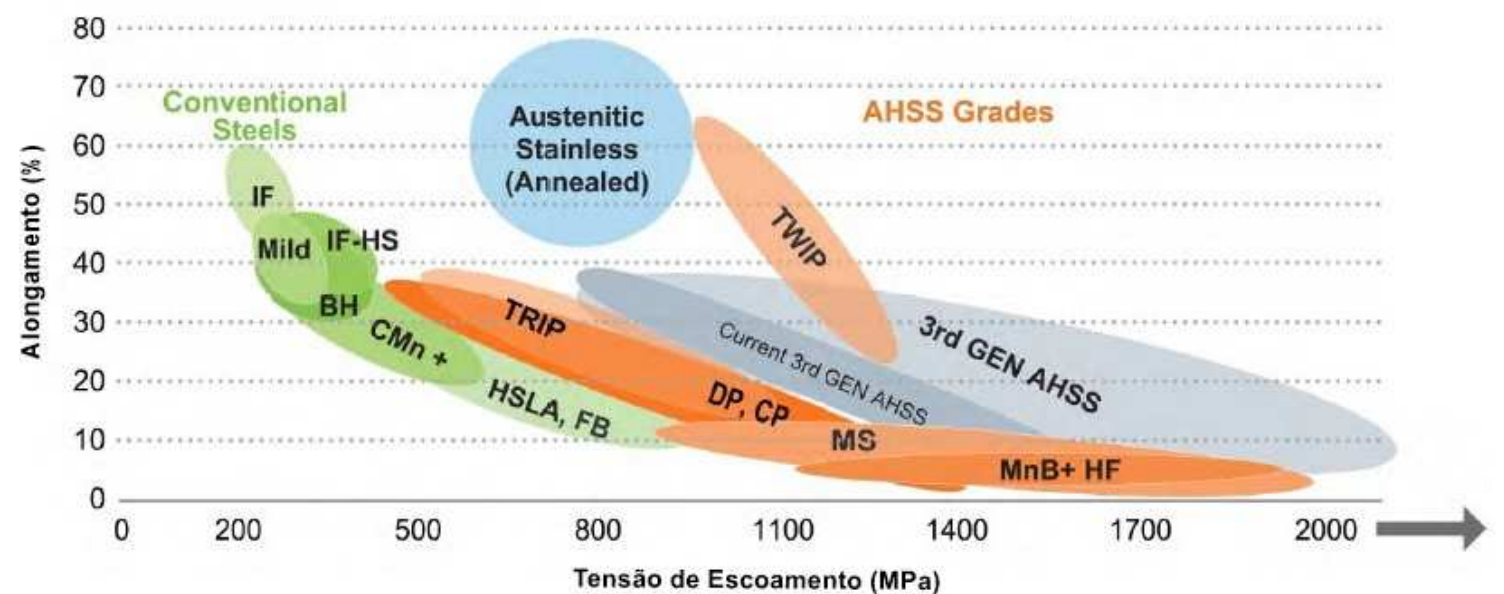

Figura 1. Comparação de propriedades mecânicas entre algumas classes de aços. Adaptada de WorldAutoSteel [2]

Os aços Dual Phase foram pioneiros na obtenção da alta resistência e ainda são extremamente utilizados na indústria. A Figura 2 apresenta a estrutura encontrada neste tipo de aço, uma faixa de 80 a $85 \%$ de ferrita macia e contínua e 15 a $20 \%$ de martensita dura em forma de ilhas isoladas localizadas nos contornos de grão da ferrita. Estas condições propiciam a alta resistência associada à ductilidade e conformabilidade que são características destes aços. Tal microestrutura permite, também, grande capacidade de encruamento ao aço, o que melhora sua capacidade de absorção de impacto e resistência à fadiga [3]. 


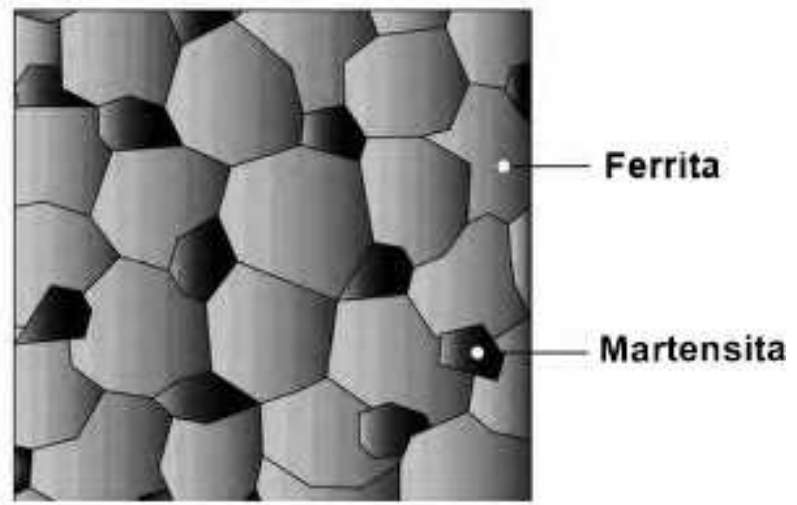

Figura 2. Micrografia esquemática de um aço Dual Phase. Adaptada de WorldAutoSteel [2]

A principal forma de produção dos aços DP é através de laminação a frio de um aço baixo carbono seguido por um recozimento intercrítico em uma linha de recozimento contínuo. A zona intercrítica se refere ao campo do diagrama Fe-C onde estão presentes as fases austenita mais ferrita. A partir destas fases prossegue-se com um resfriamento rápido para transformar a austenita restante em martensita [4].

\subsection{Ensaios de Tração e Anisotropia}

O ensaio de tração é um dos métodos mais clássicos para o conhecimento sobre as propriedades mecânicas de um material. Uma amostra é deformada, geralmente até sua ruptura, por uma carga de tração que é aumentada gradativamente e é aplicada uniaxialmente ao longo do eixo do corpo de prova. A máquina para ensaios de tração é projetada para alongar o corpo de prova a uma taxa constante, ao mesmo tempo em que mede contínua e simultaneamente a carga instantânea que está sendo aplicada e os alongamentos resultantes [5].

A curva tensão-deformação de engenharia resultante do ensaio de tração apresenta diversos conceitos e valores necessários para um trabalho com transformação mecânica, como o módulo de elasticidade, limite de escoamento, limite de resistência à tração, ductilidade, resiliência e tenacidade.

As propriedades mecânicas de um material trabalhado previamente podem variar conforme a direção em que se retira o corpo de prova para ensaio. A este fenômeno dá-se o nome de anisotropia e sua ocorrência surge devido à orientação preferencial dos grãos no metal após uma deformação mecânica ou devido ao alinhamento de inclusões, vazios, segregações ou de uma segunda fase precipitada também por trabalho mecânico.

O índice de anisotropia $R$ é definido como a razão da deformação verdadeira na largura e a deformação verdadeira na espessura no teste de um corpo de prova padrão. Para que tal deformação possa ser mensurada, o corpo de prova é submetido a um alongamento de 10 a 20\%. Após a conformação, o valor de R pode ser calculado pela expressão:

$$
R=\frac{\varepsilon_{w}}{\varepsilon_{t}}=\frac{\ln \left(\frac{w_{f}}{w_{0}}\right)}{\ln \left(\frac{t_{f}}{t_{0}}\right)}
$$

onde $w_{0}$ e $t_{0}$ são a largura e espessura iniciais e $w_{f}$ e $t_{f}$ são a largura e espessura finais, respectivamente. 


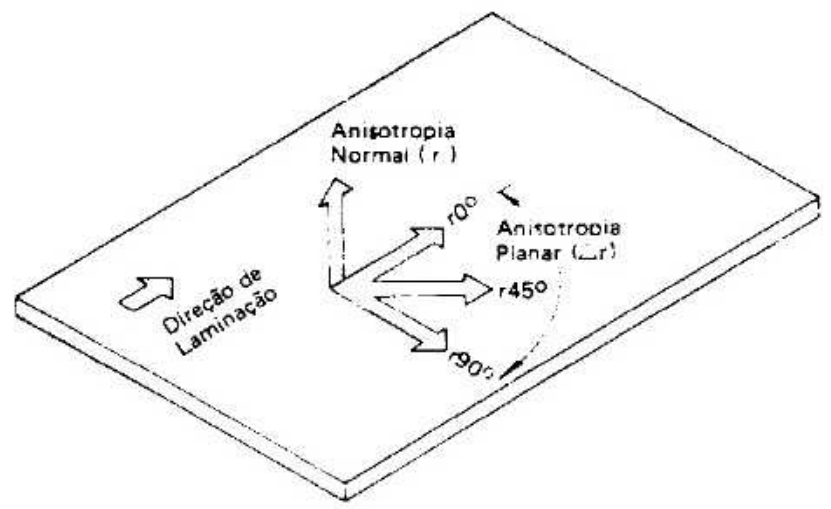

Figura 3. Desenho esquemático da orientação das direções de laminação. Adaptada de Reis [5]

O corpo de prova deve ser retirado em várias direções em relação à direção de laminação: $0^{\circ}, 45^{\circ}$ e $90^{\circ}$, como pode ser visto na Figura 3. Destes ensaios retiram-se os valores de $R_{0}, R_{45}$ e $R_{90}$. A variação de $\mathrm{R}$ dentro do plano da chapa é chamada de anisotropia planar, dada pela equação:

$$
\Delta R=\frac{R_{0}+R_{90}-2 R_{45}}{2}
$$

A principal maneira de determinação de $R$ é na direção normal à superfície da chapa laminada. O valor de $\bar{R}$ é denominado anisotropia normal ou média e é dado pela expressão:

$$
\bar{R}=\frac{R_{0}+R_{90}+2 R_{45}}{4}
$$

Se o material independe das direções de medida dentro da chapa, este é chamado isotrópico e o coeficiente $R$ tem valor 1 . Para $R>1$ ocorre uma resistência ao afinamento da chapa e, portanto, uma alta resistência da tensão biaxial. Para $R<1$ a tendência é o afinamento da chapa. Nos materiais para estampagem profunda, por exemplo, um alto valor de anisotropia normal é desejado, bem como valores baixos para a anisotropia planar, porque proporcionam maior resistência ao afinamento da chapa [3, 5-9].

Assim sendo, este trabalho tem por objetivo a caracterização de uma chapa de aço DP600 através de metalografia e quanto as suas propriedades mecânicas e anisotrópicas, comparando com valores determinados por norma e valores encontrados na literatura.

\section{MATERIAIS E MÉTODOS}

\subsection{Material Utilizado}

Para a realização deste trabalho foi utilizada uma chapa de aço DP600 de $1 \mathrm{~mm}$ de espessura. A composição química deste aço encontra-se na Tabela 1, conforme norma DIN EN 10338. De acordo com o fabricante, as mesmas especificações de composição química foram seguidas para o produto.

Tabela 1. Composição química do aço DP600. Norma DIN EN 10338

Composição Química do Aço DP600 - Valores Máximos (\%)

\begin{tabular}{cccccccccc}
\hline $\mathrm{C}$ & $\mathrm{Si}$ & $\mathrm{Mn}$ & $\mathrm{P}$ & $\mathrm{S}$ & $\mathrm{Al}$ & $\mathrm{Cr}+\mathrm{Mo}$ & $\mathrm{Nb}+\mathrm{Ti}$ & $\mathrm{V}$ & $\mathrm{B}$ \\
\hline 0,170 & 0,800 & 2,200 & 0,080 & 0,015 & 2,00 & 1,00 & 0,15 & 0,20 & 0,005 \\
\hline
\end{tabular}


Para verificar a microestrutura do material foi realizado um ensaio metalográfico. Para tal, uma pequena amostra da chapa foi cortada e embutida a quente em baquelite. Após lixamento e polimento, a amostra foi atacada com Nital $2 \%$. A análise micrográfica foi feita em microscópio óptico.

\subsection{Ensaios de Tração}

A partir da chapa de aço os corpos de prova foram cortados em guilhotina para a realização dos ensaios de tração, obedecendo as direções de $0^{\circ}$, $45^{\circ}$ e $90^{\circ}$ em relação à direção de laminação da chapa. A Figura 4 e a Tabela 2 apresentam o formato adotado para os corpos de prova e suas devidas dimensões, as quais seguem o padrão de lados paralelos, conforme as normas DIN EN 10002-1 [10] e ASTM E517-00 [11].
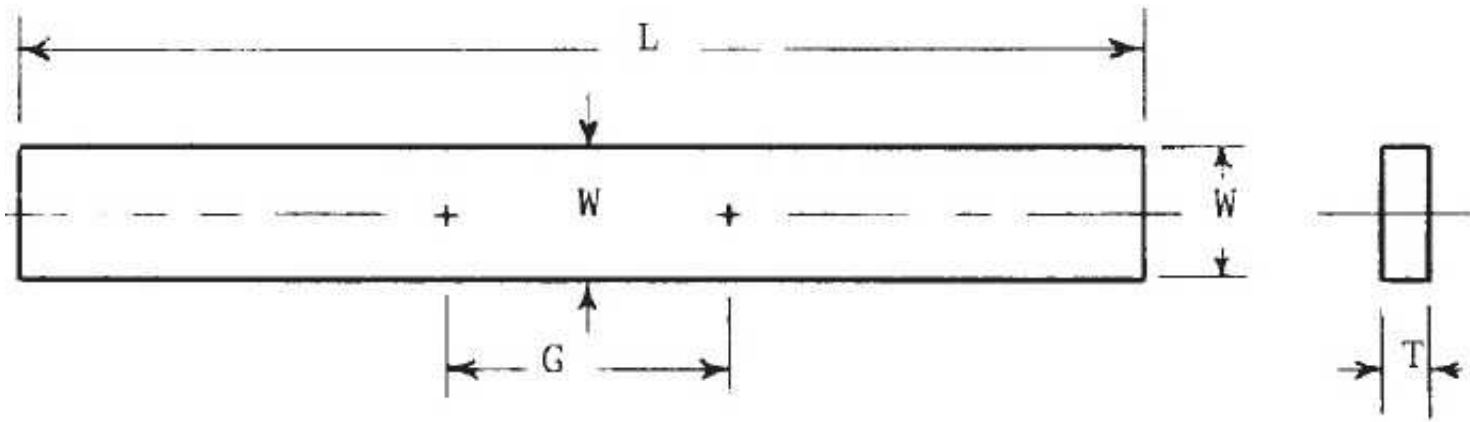

Figura 4. Forma e dimensões do corpo de prova. Adaptada de ASTM E517-00 [11]

Tabela 2. Valores padrões para as dimensões do corpo de prova

\begin{tabular}{ccc}
\hline & Método $\mathbf{B}$ - Valores Padrões & $\mathbf{m m}$ \\
\hline $\mathrm{L}$ & Comprimento Total & 200 \\
\hline $\mathrm{W}$ & Largura & $20 \pm 0,13$ \\
\hline $\mathrm{G}$ & Comprimento Útil & $50 \pm 0,25$ \\
\hline $\mathrm{T}$ & Espessura & 1 \\
\hline
\end{tabular}

Método B referido na norma ASTM E517-00 [11]

Todos os corpos de prova sofreram processo de lixamento em suas laterais com a finalidade de reduzir a incidência de rebarbas que possam levar a um acúmulo na concentração de tensões, o que reduziria a precisão dos resultados obtidos. Foram utilizados quinze corpos de prova, distribuídos para cada ensaio de acordo com a Tabela 3.

Tabela 3. Distribuição dos corpos de prova de acordo com o tipo de ensaio.

\begin{tabular}{ccc}
\hline \multirow{2}{*}{ Direção de Laminação } & \multicolumn{2}{c}{ Tipo de Ensaio } \\
\cline { 2 - 3 } & $\begin{array}{c}\text { Obtenção das Propriedades } \\
\text { Mecânicas }\end{array}$ & $\begin{array}{c}\text { Coeficientes } \\
\text { de Anisotropia }\end{array}$ \\
\hline $0^{\circ}$ & 3 & 3 \\
\hline $45^{\circ}$ & - & 3 \\
\hline $90^{\circ}$ & 3 & 3 \\
\hline
\end{tabular}


Os ensaios de tração foram realizados em um equipamento EMIC com capacidade de 60 toneladas alocado no Laboratório de Transformação Mecânica da UFRGS. Para comandar os ensaios e realizar a aferição dos dados foi utilizado o software Tesc $^{\circledR}$. O corpo de prova é preso entre duas garras do equipamento de modo que 50 $\mathrm{mm}$ de cada lado são utilizados para o agarre. Um extensômetro é posicionado nos limites do seu comprimento útil para medir a deformação sofrida. Um a um, cada corpo de prova foi ensaiado, os seis primeiros para obtenção das propriedades mecânicas e os outros nove corpos de prova para obtenção dos índices de anisotropia. Todos os valores da carga aplicada e da deformação sofrida foram obtidos em tempo real através do software, gerando ao final os dados necessários.

Os ensaios para determinação das propriedades mecânicas das chapas de aço DP600 transcorreram até a ruptura do corpo de prova, sendo que o equipamento era automaticamente desligado quando isto ocorria. Para a determinação dos índices de anisotropia, ficou determinado um alongamento máximo de $10 \%$ para o corpo de prova. Ao atingir este valor, o equipamento era desligado e o corpo de prova retirado para ser novamente medido. Todas as medições foram realizadas com paquímetro digital com tolerância dimensional de $0,01 \mathrm{~mm}$, exceto as medições de espessura inicial e final, estas realizadas com micrômetro digital de $0,001 \mathrm{~mm}$.

\section{RESULTADOS E DISCUSSÃO}

\subsection{Estrutura Metalográfica}

Através do processo de análise metalográfica da superfície da chapa, foi possível obter a visualização da microestrutura do material, conforme apresentado na Figura 5. A estrutura obtida pode ser comparada a outros trabalhos. A matriz ferrítica é caracterizada pelos grãos mais claros e envolve as ilhas de martensita de coloração mais escura.

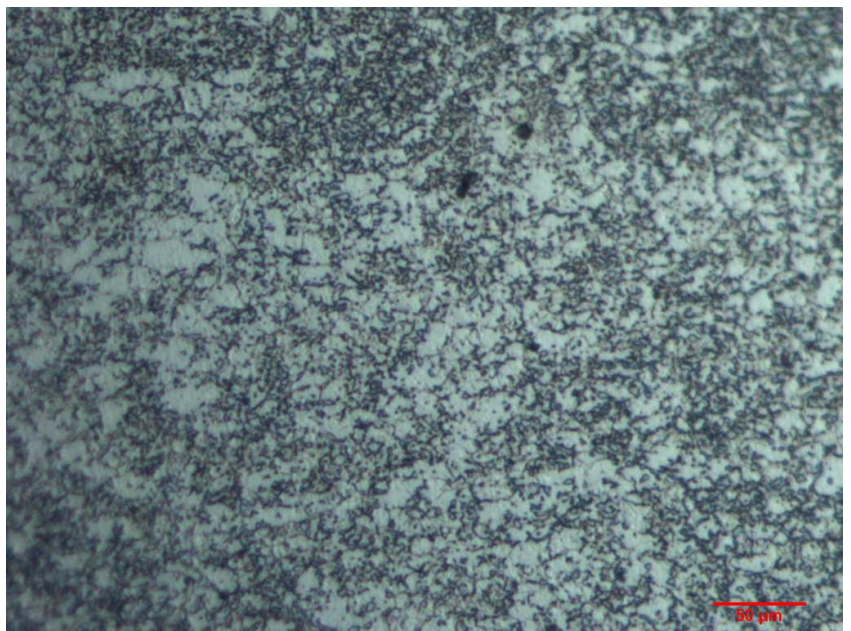

Figura 5. Micrografia do aço DP600 realizada em laboratório. Ataque com Nital 2\%. 20x.

\subsection{Propriedades Mecânicas}

A curva de escoamento reproduz o comportamento mecânico do material quando solicitado, sendo que tal comportamento irá variar conforme as diferentes direções da sua microestrutura ou coeficiente de anisotropia. Por isso, o ensaio de tração foi realizado em cada uma das amostras e os valores médios são apresentados na 
Figura 6. Pode ser observada uma diferença de aproximadamente $10 \%$ entre a direção de laminação e a direção de $90^{\circ}$ da laminação.

Para que se obtenha o comportamento mais próximo do real para a caracterização geral do material foi adotado o valor médio entre as duas direções (curva média indicada na Figura 6).

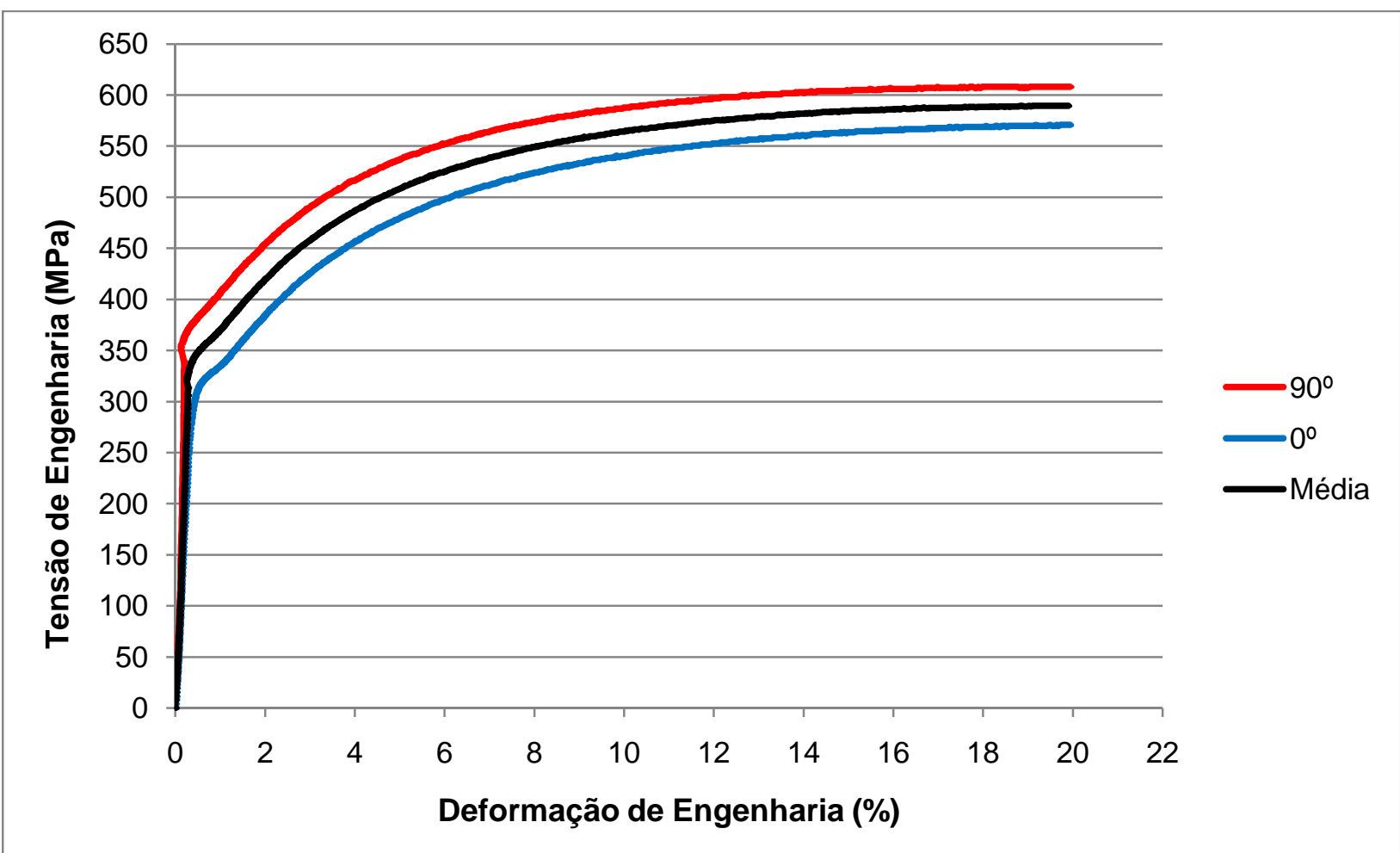

Figura 6. Curvas de tensão de engenharia versus deformação de engenharia obtidas para o aço DP600.

Os resultados mostrados na Tabela 4 são referentes à curva média comparados com os valores exigidos pela norma, os valores fornecidos pelo fabricante e os valores encontrados por Tsipouridis [12] e Tigrinho [3].

Tabela 4. Propriedades mecânicas do aço DP600

Limite de Escoamento Limite de Resistência à Tração Alongamento (MPa) (MPa) (\%)

\begin{tabular}{cccc}
\hline Ensaios de Tração & 330 & 589 & 20 \\
\hline Norma DIN EN 10338 & $340-420$ & $\geq 600$ & 20 \\
\hline Fabricante do Aço & $320-470$ & $\geq 590$ & 22 \\
\hline DP600 - Tsipouridis [12] & 380 & 660 & 23
\end{tabular}

Os melhores resultados foram encontrados para os corpos de prova retirados a $90^{\circ}$ da direção de laminação, sendo estes de $353 \mathrm{MPa}$ para o limite de escoamento e $608 \mathrm{MPa}$ para o limite de resistência à tração. O alongamento obtido foi de $20 \%$, contemplando o mínimo exigido pela norma e caracterizando a boa ductilidade do material. 
Esta variação de valores dependente da direção de laminação é esperada nos materiais que sofrem tal processo e, principalmente, em chapas finas. Alguns parâmetros podem interferir na realização dos ensaios de tração e devem ser minimizados para possibilitar um ensaio aceitável. O cuidado necessário ao trabalho com chapas finas é importante para que nenhum tipo de deformação possa ser aplicado durante o manuseio destas, como dobramento ou riscamento.

\section{3 Índices de Anisotropia}

Para calcular os índices de anisotropia foi utilizada a Equação 1 em cada corpo de prova. Os valores apresentados na Tabela 5 são uma média dos valores obtidos para cada direção de laminação. Com as Equações 2 e 3 foram realizados os cálculos de anisotropia média e anisotropia planar. Não há dados do fabricante para os coeficientes de anisotropia. Assim, a comparação foi realizada através de estudos encontrados na literatura por Tigrinho [3] e Altan et al. [13].

Tabela 5. Índices de anisotropia do aço DP600

\begin{tabular}{c|c|c|c|c|c}
\hline \multicolumn{7}{c}{ Índices de Anisotropia } \\
\hline & $\mathbf{R}_{\mathbf{0}}$ & $\mathbf{R}_{\mathbf{4 5}}$ & $\mathbf{R}_{\mathbf{9 0}}$ & $\overline{\mathbf{R}}$ & $\Delta \mathbf{R}$ \\
\hline Ensaios de Tração & 0,96 & 1,08 & 0,88 & 1,00 & $-0,17$ \\
\hline DP600 - Altan et al. [13] & 0,942 & 1,01 & 1,08 & 1,0105 & 0,001 \\
\hline DP600 - Tigrinho [3] & 0,68 & 1,04 & 1,00 & 0,94 & $-0,20$ \\
\hline
\end{tabular}

O material escoa melhor nas direções com maior índice de anisotropia. Comparando-se as direções de laminação, o maior coeficiente é encontrado a 45ำ, o que demonstra haver maior resistência ao afinamento da chapa e maior eficiência aos esforços de deformação se trabalhada nesta direção. Como todos os valores dos coeficientes $R$ são diferentes entre si e o valor da anisotropia média é 1 pode-se dizer que este aço apresenta comportamento anisotrópico planar.

Para casos de estampagem, de portas de automóveis, por exemplo, as diferentes propriedades mecânicas observadas no plano da chapa conduzem o material a sofrer um fenômeno conhecido como orelhamento. A formação de "orelhas" no produto estampado é um fato comum que deve ser evitado, já que leva à necessidade de utilização de mais material para a conformação. O orelhamento é uma consequência da anisotropia planar, sendo que quando esta for igual a zero este fenômeno não é visualizado. Para $\Delta R>0$ o orelhamento irá ocorrer em $0 \circ$ ou $90^{\circ}$. Se $\Delta R<0$, esta formação de "orelhas" ocorre próximo a 45‥ Apesar da variação observada na comparação com outros autores, estes valores ainda podem ser considerados baixos. Comparado ao valor encontrado por Altan et al. [13], a anisotropia planar calculada apresenta uma tendência maior ao orelhamento, porém em direções diferentes, enquanto que comparado a Tigrinho [3], há menor tendência ao orelhamento.

\section{CONCLUSÃO}

A microestrutura observada está em acordo com o fabricante e com os ensaios realizados por outros autores. Através dos resultados dos ensaios de tração fica comprovada a possibilidade de utilização de chapas de aço DP600 para aplicações 
a frio. A alta resistência observada e os níveis de alongamento atingidos demonstram a ductilidade característica destes aços que os faz estarem presentes em grande quantidade na estrutura de um automóvel e serem cada vez mais estudados para aplicação em outros ramos. As propriedades observadas a $90^{\circ}$ da direção de laminação se mostraram melhores, embora a diferença para com as outras direções seja pequena. Certos parâmetros como a inadequada conservação das chapas ao longo do tempo podem levar a diminuição da correlação dos resultados.

As próprias variações observadas anteriormente para os valores das propriedades mecânicas evidenciam a importância do conhecimento da anisotropia do material para que este possa ser trabalhado com o máximo de precisão e nas direções com melhor aproveitamento.

Uma continuidade deste trabalho tem por objetivo a observação do comportamento do aço DP600 em diferentes temperaturas e sua possível utilização para trabalhos a morno.

\section{Agradecimentos}

Ao CNPq pelo apoio prestado através de bolsa de iniciação científica.

\section{REFERÊNCIAS}

1 Gorni AA. Aços avançados de alta resistência: microestrutura e propriedades mecânicas. Corte \& Conformação de Metais. Dezembro, 2008; p26-57.

2 WorldAutoSteel. Advanced High Strength Steels Application Guidelines. Versão 5.0; 2014. Disponível em: www.worldautosteel.org.

3 Tigrinho LMV. Análise da fratura de chapas do aço avançado de alta resistência DP600 quando submetido a diferentes estados de tensões [Tese de Doutorado]. Curitiba: UFPR; 2011.

4 Granbom Y. Structure and mechanical properties of dual phase steels - an experimental and theoretical analysis [Tese de Doutorado]. Estocolmo: KTH Royal Institute of Technology; 2010.

5 Reis LC dos. Estudo dos parâmetros de influência na simulação numérica de estampagem de chapas [Dissertação de Mestrado]. Belo Horizonte: UFMG; 2002.

6 Callister WD Jr, Rethwisch DG. Ciência e engenharia de materiais: uma introdução. 8 Edição. Rio de Janeiro: LTC; 2013.

7 Ferrarini JL. Caracterização de materiais para o processo de estampagem [Dissertação de Mestrado]. Porto Alegre: UFRGS; 2004.

8 Srour EH Junior. Determinação do grau de conformabilidade de chapa de aço para suporte de coluna de direção e identificação de similar nacional [Dissertação de Mestrado]. Curitiba: UFPR; 2002.

9 Chemin RA Filho. Avaliação das deformações de chapas finas e curvas CLC para diferentes geometrias de punções [Dissertação de Mestrado]. Curitiba: UFPR; 2004.

10 Deutsches Institut für Normung. EN 10002-1. Metallic materials - Tensile testing - Part 1: method of test at ambient temperature. 2001.

11 American Society for Testing and Materials. E 517-00. Standard Test Method for Plastic Strain Ratio $r$ for Sheet Metal. 2000.

12 Tsipouridis P. Mechanical properties of Dual-Phase steels [Tese de Doutorado]. München: 2006.

13 Altan T, Nasser A, Yadav A, Pathak P. Determination of the flow stress of five AHSS sheet materials (DP 600, DP 780, DP 780-CR, DP 780-HY and TRIP 780) using the uniaxial tensile and the biaxial Viscous Pressure Bulge (VPB) tests. Journal of Materials Processing Technology. 2010; 210:429-436. 